\title{
The Lippmann-Schwinger Formula and One Dimensional Models with Dirac Delta Interactions
}

\begin{abstract}
We show how a proper use of the Lippmann-Schwinger equation 5 simplifies the calculations to obtain scattering states for one dimensional systems 6 perturbed by $N$ Dirac delta equations. Here, we consider two situations. In the 7 former, attractive Dirac deltas perturbed the free one dimensional Schrödinger 8 Hamiltonian. We obtain explicit expressions for scattering and Gamow states. For 9 completeness, we show that the method to obtain bound states use comparable 10 formulas, although not based on the Lippmann-Schwinger equation. Then, the 11 attractive $N$ deltas perturbed the one dimensional Salpeter equation. We also obtain 12 explicit expressions for the scattering wave functions. Here, we need regularisation 13 techniques that we implement via heat kernel regularisation.

Keywords Scattering states $\cdot$ Schrödinger and Salpeter one dimensional 15 Hamiltonians · Contact perturbations · Gamow wave functions · 16 $\begin{array}{ll}\text { Lippmann-Schwinger equation } & 17\end{array}$
\end{abstract}

\section{Introduction}

One of the more used tools in order to understand quantum mechanics are the 19 solvable models, in particular those which are one dimensional due to their 20 simplicity [1-4]. The more often studied among these models is the free particle 21 Schrödinger Hamiltonian decorated with Dirac delta interactions. Relativistic one 22

\section{F. Erman}

Department of Mathematics, Izmir Institute of Technology, Urla, Izmir, Turkey e-mail: fatih.erman@gmail.com

\section{Gadella $(\bowtie)$}

Departamento de Física Teórica, Atómica y Óptica and IMUVA, Universidad de Valladolid, Valladolid, Spain

H. Uncu

Department of Physics, Adnan Menderes University, Aydın, Turkey

e-mail: huncu@adu.edu.tr 
dimensional approaches for the free particle Hamiltonian, such as those named after 23 Salpeter or Dirac, have also been perturbed with contact interactions of delta type 24 [5-7]. The purpose of the present article is to give a brief review of the recent work 25 by the authors including the perturbation by $N$ Dirac deltas of the one dimensional 26 Schrödinger and Salpeter free Hamiltonians $[6,8,9]$.

From the physics point of view, point potentials may represent interactions which 28 are very localised in the space and strong and have a vast amount of applications for 29 modelling real physical systems. A well-known model using Dirac delta potentials 30 in non-relativistic quantum mechanics is the so-called Kronig-Penney model [10], 31 and it is actually a reference model in describing the band gap structure of metals 32 in solid state physics [11]. In addition, Dirac delta interactions in one or more 33 dimensions serve as simple pedagogical toy models for the understanding of several 34 quantum non-trivial concepts [12-19].

From the mathematical point of view, contact potentials are the result of the 36 theory of self-adjoint extensions of symmetric operators with equal deficiency 37 indices. In general, there are two methods to obtain these extensions. One is by 38 defining some matching conditions at the nodes (points that support the contact 39 potentials). Other uses the construction of the resolvent operator and often requires 40 a renormalisation due to possible divergences in the construction of the resolvent of 41 the self-adjoint extension. Still a third method relies on a theorem of von Neumann 42 that characterises all self-adjoint extensions of a symmetric operator with equal 43 deficiency indices, although this one has been less used.

We also want to show how the Lippmann-Schwinger formula is useful for 45 this purpose as a simplifying computational tool. Here, we shall use the simplest 46 form of this equation which acquires mathematical sense on Gelfand triplets. The 47 Lippmann-Schwinger formula gives an equation satisfied by the incoming and 48 outgoing plane waves after a scattering process due to a potential $V$. It has the 49 following form:

$$
\left|k^{ \pm}\right\rangle=|k\rangle-R_{0}\left(E_{k} \pm i 0\right) V\left|k^{ \pm}\right\rangle
$$

where $\left|k^{ \pm}\right\rangle$refers to the full scattered incoming (+) and outgoing (-) plane waves, 51 $|k\rangle$ is the free plane wave, $V$ the potential and $R_{0}\left(E_{k} \pm i 0\right)$ is the free resolvent, also 52 called the Green operator. Since it is a function of the complex variable $z, R(z)$, and 53 has a branch cut at the spectrum of the free Hamiltonian (usually $\mathbb{R}^{+} \equiv[0, \infty)$ ), we 54 denote by $R_{0}\left(E_{k} \pm i 0\right)$ the upper and lower limits of $R(z)$ as the imaginary part of 55 $z$ goes to zero. Here, $E_{k}=\left(\hbar^{2} k^{2}\right) / 2 m$.

This paper contains three more sections. In Sect.2, we briefly discuss the 57 consequences of adding $N$ Dirac delta perturbations to the one dimensional free 58 Schrödinger Hamiltonian. In Sect.3, we do the same with the one dimensional 59 Salpeter Hamiltonian. The analysis of bound states is particularly relevant in both 60 cases. We finish our discussion with the concluding remarks. 


\section{One Dimensional Schrödinger Hamiltonian with $N$ Dirac Delta Interactions}

The objective of this section is to study the one dimensional Schrödinger Hamil- 64 tonian $H_{0}=\frac{p^{2}}{2 m}$ perturbed by $N$ Dirac deltas located at some points in the real 65 axis. This study includes the search for bound states, scattering coefficients and 66 resonances provided they exist. As is well known, this perturbed Hamiltonian has 67 the form

$$
H:=\frac{p^{2}}{2 m}-\sum_{i=1}^{N} \lambda_{i} \delta\left(x-a_{i}\right), \quad V:=-\sum_{i=1}^{N} \lambda_{i} \delta\left(x-a_{i}\right),
$$

where $\lambda_{i}$ and $i=1,2, \ldots, N, i=1,2, \ldots, N$ are positive real numbers. The $a_{i} 69$ show the points supporting the deltas and are called nodes. Each of the $-\lambda_{i}$, with 70 $\lambda_{i}>0$, is the intensity of the delta located at $a_{i}$ for all value of $i$. These coefficients 71 are chosen to be negative if we want to have bound states. The Schrödinger equation 72 produced by (2) is

$$
-\frac{\hbar^{2}}{2 m} \frac{d^{2} \psi(x)}{d x^{2}}-\sum_{i=1}^{N} \lambda_{i} \delta\left(x-a_{i}\right) \psi(x)=E \psi(x) .
$$

It is interesting to rewrite the interaction $V$ in such a way that the calculations 74 with the aid of the Lippmann-Schwinger equation become easy. For simplicity, let 75 us assume that we have only one first. Then, the potential is $V=\lambda \delta(x-a)$ and the 76 wave function is $\psi(x)=\langle x \mid \psi\rangle$ [20-24]. In this notation, $(V \psi)(x)=\langle x \mid V \psi\rangle$ and 77 $\langle x \mid a\rangle=\delta(x-a)$. Thus,

$$
(V \psi)(x)=\lambda \delta(x-a) \psi(a) .
$$

Next, we note that the potential can be written as $V=\lambda|a\rangle\langle a|$, since then,

$$
\langle x \mid V \psi\rangle=\lambda\langle x \mid a\rangle\langle a \mid \psi\rangle=\lambda \delta(x-a) \psi(a)=(V \psi)(x) .
$$

The generalisation of the expression for the potential $V$ in the case of having $N 80$ nodes is the following:

$$
V=-\sum_{i=1}^{N} \lambda_{i}\left|a_{i}\right\rangle\left\langle a_{i}\right|
$$

This is the desired expression. Let us clarify the vectors $|x\rangle$ for any real number 82 $x$ are the generalised eigenvalues of the position (multiplication) operator in one 83 dimension with eigenvalue $x$. As is well known, these vectors do not belong to the 84 
Hilbert space on which the multiplication operator acts, but instead to an extension 85 of it endowed with a weak topology. We do not want to enter in these kind of details 86 here, see [21-24]. Vectors $\left|a_{i}\right\rangle$ are precisely of this type with $x=a_{i} . \quad 87$

The first objective is the search for scattering states. We are introducing the 88 procedure in the sequel, although we shall skip some steps in order to reach the 89 final result as straightforward as possible. Details may be found in [8,9]. Let us 90 use (6) in the Lippmann-Schwinger equation (1) and multiply the result from the 91 left by the bra $\langle x|$. We have

$$
\left\langle x \mid k^{ \pm}\right\rangle=\langle x \mid k\rangle+\sum_{j=1}^{N} \lambda_{j}\left\langle x\left|G_{0}\left(E_{k} \pm i 0\right)\right| a_{j}\right\rangle\left\langle a_{j} \mid k^{ \pm}\right\rangle .
$$

For convenience, we shall use the notation $G_{0}\left(x, y ; E_{k} \pm i 0\right):=\langle x| G_{0}\left(E_{k} \pm 93\right.$ $i 0)|y\rangle$ in the sequel. Also, we recall that $\langle x \mid k\rangle$ is the free plane wave and 94 $\psi_{k}^{ \pm}(x):=\left\langle x \mid k^{ \pm}\right\rangle$the perturbed plane wave in the coordinate representation. In 95 consequence, (7) can be written as (Henceforth we shall consider the sign plus in (7) 96 only, for simplicity. Similar results would be obtained with the other choice.)

$$
\psi_{k}^{+}(x)=e^{i k x}+\sum_{j=1}^{N} \lambda_{j} G_{0}\left(x, a_{j} ; E_{k}+i 0\right) \psi_{k}^{+}\left(a_{j}\right),
$$

The goal is now to obtain the explicit form of $\psi^{+}(x)$, for which we have to find 98 the explicit form of the terms under the sum in (8). First, let as choose as values 99 of $x$ in (8) the $\left\{a_{j}\right\}$. We obtain the following linear system of $N$ equations for $N 100$ indeterminates:

$$
\begin{aligned}
e^{i k a_{i}}= & \psi^{+}\left(a_{i}\right)\left[1-\lambda_{i} G_{0}\left(a_{i}, a_{i} ; E_{k}+i 0\right)\right] \\
& -\sum_{j \neq i}^{N} \lambda_{j} G_{0}\left(a_{i}, a_{j} ; E_{k}+i 0\right) \psi^{+}\left(a_{j}\right), \quad i=1,2, \ldots, N .
\end{aligned}
$$

This system can be rewritten in matrix form. If $\Phi \equiv\left\{\Phi_{i j}\right\}$ is the $N \times N$ matrix 102 with matrix elements

$$
\Phi_{i j}\left(E_{k}+i 0\right)=\left\{\begin{array}{c}
1-\lambda_{i} G_{0}\left(a_{i}, a_{i} ; E_{k}+i 0\right) \text { if } i=j, \\
\lambda_{j} G_{0}\left(a_{i}, a_{j} ; E_{k}+i 0\right) \text { if } i \neq j .
\end{array}\right.
$$

Then, Eqs. (9) take the form,

$$
\sum_{j=1}^{N} \Phi_{i j}\left(E_{k}+i 0\right) \psi_{k}^{+}\left(a_{j}\right)=e^{i k a_{j}}, \quad j=1,2, \ldots, N
$$


with solution,

$$
\psi_{k}^{+}\left(a_{j}\right)=\sum_{j=1}^{N}\left[\Phi^{-1}\left(E_{k}+i 0\right)\right]_{i j} e^{i k a_{j}}
$$

where $\Phi^{-1}$ is the inverse of the matrix $\Phi$. In consequence, the final form of (8) is

$$
\psi_{k}^{+}(x)=e^{i k x}+\sum_{j=1}^{N} \lambda_{j} G_{0}\left(x, a_{j} ; E_{k}+i 0\right)\left[\Phi^{-1}\left(E_{k}+i 0\right)\right]_{i j} e^{i k a_{j}}
$$

Then, we have to find the Green function $G_{0}\left(x, a_{j} ; E_{k}+i 0\right)$. We do not intend 107 to describe the procedure here, which is explained in detail in [9]. Once we have 108 obtained this Green function, using (10), we finally get all matrix elements of $\Phi .109$ The final results are

$$
G_{0}\left(x, a_{j} ; E_{k}+i 0\right)=\frac{i m}{\hbar^{2} k} e^{k\left|x-a_{i}\right|}
$$

and

$$
\Phi_{i j}\left(E_{k}+i 0\right)=\left\{\begin{array}{clr}
1-\frac{i m \lambda_{i}}{\hbar^{2} k} & \text { if } & i=j \\
-\sqrt{\lambda_{i} \lambda_{j}} \frac{i m}{\hbar^{2} k} e^{i k\left|a_{i}-a_{j}\right|} & \text { if } & i \neq j
\end{array}\right.
$$

Then, we have determined all the perturbed plane waves $\psi_{k}^{+}(x)$. For $\psi_{k}^{-}(x)$, we 112 follow a similar procedure. Always recall that $E_{k}=\left(\hbar^{2} k^{2}\right) / 2 m$.

\subsection{Search for Bound States}

So far, we have found the scattering states corresponding to the total (or perturbed) 115 Hamiltonian, for which we have used the Lippmann-Schwinger equation as main 116 tool. Next, we search for the possible existence of bound states, where the search 117 could be carried out with similar tools to those used in the precedent discussion. 118

We proceed as follows: Let us use the simplified notation $\left|f_{i}\right\rangle:=\sqrt{\lambda_{i}}\left|a_{i}\right\rangle$, so 119 that the total Hamiltonian (2) may be written as

$$
H=\frac{p^{2}}{2 m}-\sum_{i=1}^{N}\left|f_{i}\right\rangle\left\langle f_{i}\right| .
$$

The corresponding Schrödinger equation reads 


$$
\left\langle x\left|\frac{p^{2}}{2 m}\right| \psi\right\rangle-\sum_{i=1}^{N}\left\langle x \mid f_{i}\right\rangle\left\langle f_{i} \mid \psi\right\rangle=E\langle x \mid \psi\rangle
$$

Bound states correspond to solutions of (17) with negative $E$ and square integrable 122 wave function $\psi(x) \equiv\langle x \mid \psi\rangle$.

Next, insert the completeness relation $1=\frac{1}{2 \pi \hbar} \int|p\rangle\langle p| d p$ in front of $|\psi\rangle$ and 124 $\left|f_{i}\right\rangle$. Define $\widetilde{\psi}(p):=\langle p \mid \psi\rangle$, which is indeed the Fourier transform of $\langle x \mid \psi\rangle$, and 125 write $\phi\left(a_{i}\right):=\left\langle f_{i} \mid \psi\right\rangle=\sqrt{\lambda_{i}}\left\langle a_{i} \mid \psi\right\rangle=\sqrt{\lambda_{i}} \psi\left(a_{i}\right)$. Recall that $\langle x \mid p\rangle=e^{\frac{i}{\hbar} p x} .126$ Then, (17) becomes

$$
\int_{-\infty}^{\infty} \frac{d p}{2 \pi \hbar} e^{\frac{i}{\hbar} p x} \widetilde{\psi}(p)\left(\frac{p^{2}}{2 m}-E\right)=\sum_{i=1}^{N} \sqrt{\lambda_{i}} \int_{-\infty}^{\infty} \frac{d p}{2 \pi \hbar} e^{\frac{i}{\hbar} p\left(x-a_{i}\right)} \phi\left(a_{i}\right) .
$$

From (18) and the properties of the Fourier transform, we have that

$$
\widetilde{\psi}(p)=\sum_{i=1}^{N} \sqrt{\lambda_{i}} \frac{e^{-\frac{i}{\hbar} p a_{i}}}{\frac{p^{2}}{2 m}-E} \phi\left(a_{i}\right) .
$$

But $\widetilde{\psi}(p)$ is the Fourier transform of the solution $\psi(x)$ of the Schrödinger 129 equation (17). Let us use this idea to conclude that (take $x=a_{i}$ )

$$
\psi\left(a_{i}\right)=\sum_{i=1}^{N} \sqrt{\lambda_{i}} \int_{-\infty}^{\infty} \frac{d p}{2 \pi \hbar} \frac{e^{-\frac{i}{\hbar} p a_{i}}}{\frac{p^{2}}{2 m}-E} \phi\left(a_{i}\right)
$$

Multiply both sides in (20) by $\sqrt{\lambda_{i}}$ and recalling that $\phi\left(a_{i}\right)=\sqrt{\lambda_{i}} \psi\left(a_{i}\right)$, we 131 arrive to an equation of the form:

$$
\sum_{j=1}^{N} \Phi_{i j}(E) \phi\left(a_{j}\right)=0
$$

Find details in [8]. It is beyond a mere coincidence that the matrix elements $\Phi \equiv 133$ $\left\{\Phi_{i j}(E)\right\}$ are identical to those of (15) with the replacement $k=\sqrt{2 m|E|}$, so that [8] 134

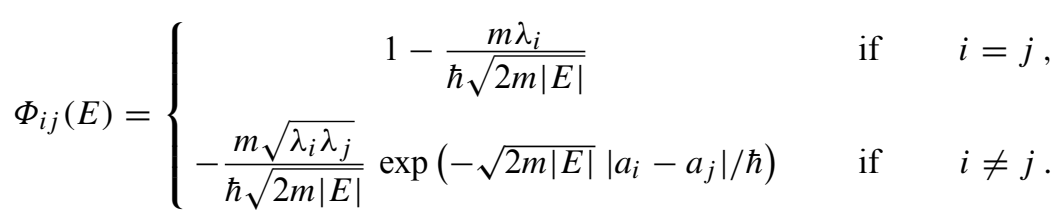

Since Eq. (21) has come directly from (17), it is a necessary condition for the 135 existence of solutions of (17) with the desired properties. This equation has non- 136 
trivial solutions $\left\{\phi\left(a_{j}\right)\right\}$ if and only if $\operatorname{det} \Phi(E)=0$. Therefore, the bound states 137 energies are solutions of the transcendental equation $\operatorname{det} \Phi(E)=0 .{ }^{1}$

For a systematic calculation of the bound states, let us consider the following 139 eigenvalue problem:

$$
\Phi(E) A(E)=\omega(E) A(E)
$$

where $\omega(E)$ are the eigenvalues of the $N \times N$ matrix $\Phi(E)$ and $A(E)$ their 141 corresponding eigenvectors. Equations (21) and (23) coincide if and only if $\omega(E)=142$ 0 and then, the bound states energies have to be the solutions of the transcendental 143 equation $\omega(E)=0$ its eigenvectors being those with components equal to $\phi\left(a_{j}\right) .144$ If we assume no degeneracy, the wave function corresponding to the energy value 145 $E_{i}$ with eigenvector $A(E) \equiv\left(\phi\left(a_{1}\right), \ldots, \phi\left(a_{N}\right)\right)$ takes the form (19) with $E=E_{i} . \quad 146$ In the coordinate representation, the wave function is just its Fourier transform. For 147 further comments, see $[6,8]$.

\subsection{Resonances and Gamow States}

The Lippmann-Schwinger equation is also useful for the construction of Gamow 150 states, which are vector states for resonances. In a resonant scattering process [25] 151 produced by a Hamiltonian pair, say $\left\{H_{0}, H\right\}$, where $H_{0}$ is a free Hamiltonian and 152 $H=H_{0}+V$, where $V$ is the interaction, the Gamow vectors, $\psi^{ \pm}$, for a resonance 153 with energy $E_{R}$ and inverse of the mean life given by $\Gamma$ are two eigenvectors of $H \quad 154$ with respective eigenvalues $E_{R} \pm \Gamma / 2$, i.e., $H \psi^{ \pm}=\left(E_{R} \mp \Gamma / 2\right) \psi^{ \pm}[25]$. This 155 property shows that the Gamow vector $\psi^{+}$decays exponentially as $t \longmapsto \infty$ (and 156 $\psi^{-}$decays exponentially as $t \longmapsto-\infty$, they are time reversal of each other). This 157 situation produces two problems, one from the point of view of physics and the other 158 from the point of view of mathematics. 159

Although exponential decay for simple quantum unstable systems has been 160 detected for essentially for all values of time, deviations for these exponential law 161 have been detected for very short or very large times [26,27]. Since these deviations 162 certainly occur under these conditions only, they are very difficult to be detected. For 163 most values of time, exponential decay serves as an excellent approximation. This 164 is why Gamow vectors are useful as good approximations of decaying states. $\quad 165$

A self-adjoint operator on Hilbert space, as is the case of the Hamiltonian $H, 166$ cannot have complex eigenvalues with corresponding eigenvectors in this Hilbert 167 space. Thus, Gamow vectors are well-defined objects on some extensions of Hilbert 168 spaces called rigged Hilbert spaces [25, 28-30].

\footnotetext{
${ }^{1}$ As a matter of fact, this also follows because $\Phi(E)$ appears in the denominator of the resolvent of the total Hamiltonian $H$.
} 
Let us briefly sketch the use of (1) to obtain an explicit expression of the Gamow 170 vectors as eigenvectors of $H$ with eigenvalue $E_{R} \pm \Gamma / 2$. Details may be found 171 in $[9,31]$. If we multiply Eq. (1) to the right by the bra $\langle\psi|$, we obtain a complex 172 function on the variable $k$. With adequate choices of the space of bras, this results on 173 meromorphic functions of complex variable defined at least on a half plane [29,30]. 174 Let us assume that this is the case and omit the bra $\langle\psi|$. Then, if we define $k_{R}$ as ${ }_{175}$

$$
z_{R}:=E_{R}-\Gamma / 2=\frac{k_{R}^{2} \hbar^{2}}{2 m},
$$

we may consider the analytic extension of (1) to the value of $k$ given by $k_{R}$,

$$
\left|k_{R}^{+}\right\rangle=\left|k_{R}\right\rangle-G_{0}\left(z_{R}\right) V\left|k_{R}^{+}\right\rangle \text {. }
$$

It is important to remark that $z_{R}$ is a pole of the Green function corresponding to the 177 total Hamiltonian $H$, but not of the free Hamiltonian $H_{0}$, just by the characterisation 178 of resonances using the resolvent [32]. Then, $G_{0}\left(z_{R}\right)$ is well defined and so is $\left|k_{R}^{+}\right\rangle, \quad 179$ which has the property $[9,31,33]$

$$
H\left|k_{R}^{+}\right\rangle=z_{R}\left|k_{R}^{+}\right\rangle
$$

Thus, $\left|k_{R}^{+}\right\rangle$is one of the Gamow vectors with resonance pole $z_{R}$ (the other can be 181 obtained exactly in the same way, just replacing $z_{R}$ by its complex conjugate $z_{R}^{*}$ and 182 taking the minus sign in (1). This Gamow vector in the coordinate representation is 183 $\psi_{R}^{+}(x):=\left\langle x \mid k_{R}^{+}\right\rangle$, so that

$$
\left(H \psi_{R}^{+}\right)(x)=\left\langle x|H| k_{R}^{+}\right\rangle=z_{R}\left\langle x \mid k_{R}^{+}\right\rangle=z_{R} \psi_{R}^{+}(x) .
$$

Now, let us go back to the $N$ Dirac deltas interaction and, consequently, take 185 in (25) the form of the potential given by $V=-\sum_{i=1}^{N} \lambda_{i}\left|a_{i}\right\rangle\left\langle a_{i}\right|$. Multiply the 186 result of this operation to the right by the bra $\langle x|$ and divide $k_{R}$ into real and 187 imaginary parts, $k_{R}=k_{r}-i k_{I}$. We have that $\left\langle x \mid k_{R}\right\rangle=e^{i k_{R} x}=e^{i k_{r} x} e^{-i k_{I} x}$ and $\quad 188$

$$
\begin{aligned}
\psi_{k}^{+}(x)= & \left\langle x \mid k_{R}^{+}\right\rangle=\left\langle x \mid k_{R}\right\rangle+\sum_{i=1}^{N} \lambda_{i}\left\langle x\left|G_{0}\left(z_{R}\right)\right| a_{i}\right\rangle\left\langle a_{i} \mid k_{R}^{+}\right\rangle \\
= & e^{i k_{r} x} e^{k_{I} x}+\sum_{i=1}^{N} \lambda_{i} G_{0}\left(x, a_{i} ; z_{R}\right) \psi_{R}^{+}\left(a_{i}\right)=e^{i k_{r} x} e^{k_{I} x} \\
& +\sum_{i=1}^{N} \lambda_{i} \sum_{j=1}^{N} \frac{i m \sqrt{\lambda_{i} \lambda_{j}}}{\hbar^{2}\left(k_{r}-i k_{I}\right)}\left[e^{i\left(k_{r}-i k_{I}\right)\left|x-a_{i}\right|} \Phi^{-1}\left(z_{R}\right)\right]_{i j} e^{i\left(k_{r}-i k_{I}\right) a_{j}} .
\end{aligned}
$$


A similar result can be obtained for the Gamow wave function $\psi^{-}(x)$. In 189 principle, both Gamow functions will be equally suitable to play the role of wave 190 function for the resonance state. The only technical difference is that one represents 191 the time reversal of the other [30]. Observe that $\psi_{k}^{+}(x) \longmapsto \infty$ as $x \longmapsto \infty .{ }^{192}$ Gamow wave functions cannot be normalised in the usual sense of square integrable 193 normalisation, but in sharp contrast with the plane waves (Dirac kets) which are not 194 normalisable although bounded, Gamow functions show an exponential growing at 195 the spatial infinite. This behaviour has been often called the exponential catastrophe. 196 This is not such a problem with a proper interpretation of the Gamow wave function 197 in terms of generalised functions in a suitable rigged Hilbert space. Still, this expo- 198 nential behaviour creates some particular problems such as the difficulties arisen in 199 order to fix a proper definition of averages of observables in Gamow states [34, 35]. 200

\section{One Dimensional Salpeter Hamiltonian with $N$ Deltas}

The one dimensional Salpeter Hamiltonian decorated with $N$ Dirac deltas has the 202 following form $(c=1)$ :

$$
H:=\sqrt{p^{2}+m^{2}}-\sum_{i=1}^{N} \lambda_{i} \delta\left(x-a_{i}\right), \quad H_{0}:=\sqrt{p^{2}+m^{2}} .
$$

Here, $H_{0}$ is the free Salpeter Hamiltonian. The definition of a self-adjoint version 204 for $H$ in (29) is not as simple as is in the Schrödinger case, where it is sufficient to 205 impose correct matching conditions at the nodes. This self-adjoint version is usually 206 determined by a proper choice of the resolvent operator of $H$, which should be 207 obtained from the resolvent operator of $H_{0}$ by the Krein formula. However, this 208 procedure leads to divergences in our case, so that a regularisation procedure is 209 in order here $[5,6]$. We have chosen heat kernel regularisation for several reasons 210 discussed in [6]. Let us sketch briefly the procedure. First of all, we write the 211 Hamiltonian $H$ as in (29) as

$$
H=\sqrt{p^{2}+m^{2}}-\sum_{i=1}^{N} \lambda_{i}\left|a_{i}\right\rangle\left\langle a_{i}\right|,
$$

exactly as we did for the cases studied in the previous section. The next step is to 213 write an $\epsilon$-regularised version of (30) as

$$
H_{\epsilon}=\sqrt{p^{2}+m^{2}}-\sum_{i=1}^{N} \lambda_{i}(\epsilon)\left|a_{i}^{\epsilon}\right\rangle\left\langle a_{i}^{\epsilon}\right|
$$


where the new kets $\left|a_{i}^{\epsilon}\right\rangle$ are defined in such a way that $\left\langle x \mid a_{i}^{\epsilon}\right\rangle:=K_{\epsilon / 2}\left(x, a_{i}\right)$, where 215 the function $K_{t}(x, y)$ is the so-called heat kernel, which is the fundamental solution 216 of the heat equation of the form:

$$
\sqrt{p^{2}+m^{2}} K_{t}(x, y)=-\frac{\partial K_{t}(x, y)}{\partial t},
$$

and the weights $\lambda(\epsilon)$ are also chosen as functions of the parameter $\epsilon$, such that 218 $\lim _{\epsilon \rightarrow 0^{+}} \lambda_{i}(\epsilon) \longmapsto \lambda_{i}, i=1,2, \ldots, N$. The interest of this choice for $\left\langle x \mid a_{i}^{\epsilon}\right\rangle$ comes 219 after the limiting property $\left\langle x \mid a_{i}^{\epsilon}\right\rangle \longmapsto\left\langle x \mid a_{i}\right\rangle=\delta\left(x-a_{i}\right)$ as $\epsilon \longmapsto 0^{+}$.

Now, we go back to the Lippmann-Schwinger equation (1), where in the present 221 case $E_{k}=\sqrt{p^{2}+m^{2}}$ and $V$ is as in (31). This gives

$$
\left|k^{ \pm}(\epsilon)\right\rangle=|k\rangle+\sum_{j=1}^{N} \lambda_{j}(\epsilon) R_{0}\left(E_{k} \pm i 0\right)\left|a_{j}^{\epsilon}\right\rangle\left\langle a_{j}^{\epsilon} \mid k^{ \pm}\right\rangle .
$$

Let us choose the plus sign in (33) and use for brevity the following notation: 223 $\left|f_{i}^{\epsilon}\right\rangle:=\sqrt{\lambda_{i}(\epsilon)}\left|a_{i}^{\epsilon}\right\rangle$. Then, we choose one subindex $i$ and isolate the corresponding 224 term in (33):

$$
\begin{aligned}
\left|k^{+}(\epsilon)\right\rangle= & |k\rangle+R_{0}\left(E_{k}+i 0\right)\left|f_{i}^{\epsilon}\right\rangle\left\langle f_{i}^{\epsilon} \mid k^{+}(\epsilon)\right\rangle \\
& +\sum_{j \neq i}^{N} R_{0}\left(E_{k}+i 0\right)\left|f_{j}^{\epsilon}\right\rangle\left\langle f_{j}^{\epsilon} \mid k^{+}(\epsilon)\right\rangle,
\end{aligned}
$$

before multiplying (34) to the left by the ket $\left\langle f_{i}^{\epsilon}\right|$. This gives

$$
\begin{aligned}
& {\left[1-\left\langle f_{i}^{\epsilon}\left|R_{0}\left(E_{k}+i 0\right)\right| f_{i}^{\epsilon}\right\rangle\right]\left\langle f_{i}^{\epsilon} \mid k^{+}(\epsilon)\right\rangle} \\
& -\sum_{i \neq j}^{N}\left[\left\langle f_{i}^{\epsilon}\left|R_{0}\left(E_{k}+i 0\right)\right| f_{i}^{\epsilon}\right\rangle\right]\left\langle f_{i}^{\epsilon} \mid k^{+}(\epsilon)\right\rangle=\left\langle f_{i}^{\epsilon} \mid k\right\rangle,
\end{aligned}
$$

expression valid for $i=1,2, \ldots, N$. This may be written in the matrix form as

$$
\sum_{j=1}^{N} T_{i j}\left(\epsilon, E_{k}+i 0\right)\left\langle f_{j}^{\epsilon} \mid k^{+}(\epsilon)\right\rangle=\left\langle f_{i}^{\epsilon} \mid k\right\rangle, \quad j=1,2, \ldots, N
$$

with

$$
T_{i j}\left(\epsilon, E_{k}+i 0\right)=\left\{\begin{array}{lll}
1-\left\langle f_{i}^{\epsilon}\left|R_{0}\left(E_{k}+i 0\right)\right| f_{i}^{\epsilon}\right\rangle & \text { if } \quad i=j \\
-\left\langle f_{i}^{\epsilon}\left|R_{0}\left(E_{k}+i 0\right)\right| f_{j}^{\epsilon}\right\rangle & \text { if } \quad i \neq j
\end{array}\right.
$$


Therefore, we may write the solution of (36) as

$$
\left\langle f_{i}^{\epsilon} \mid k^{+}(\epsilon)\right\rangle=\sum_{j=1}^{N}\left[T^{-1}\left(\epsilon, E_{k}+i 0\right)\right]_{i j}\left\langle f_{j}^{\epsilon} \mid k\right\rangle
$$

We use (38) in (35) and, then, multiply the result to the left by the bra $\langle x|$. This gives

$$
\begin{aligned}
\psi^{+}(\epsilon, x) & :=\left\langle x \mid k^{+}(\epsilon)\right\rangle \\
& =\langle x \mid k\rangle+\sum_{i, j=1}^{N}\left\langle x\left|R_{0}\left(E_{k}+i 0\right)\right| f_{i}^{\epsilon}\right\rangle\left[T^{-1}\left(\epsilon, E_{k}+i 0\right)\right]_{i j}\left\langle f_{j}^{\epsilon} \mid k\right\rangle \\
& =e^{i k x}+\sum_{i, j=1}^{N}\left\langle x\left|R_{0}\left(E_{k}+i 0\right)\right| a_{i}^{\epsilon}\right\rangle\left[\Phi^{-1}\left(\epsilon, E_{k}+i 0\right)\right]_{i j}\left\langle a_{j}^{\epsilon} \mid k\right\rangle,
\end{aligned}
$$

with

$$
\Phi_{i j}\left(\epsilon, E_{k}+i 0\right)=\left\{\begin{array}{clr}
\frac{1}{\lambda_{i}(\epsilon)}-\left\langle a_{i}^{\epsilon}\left|R_{0}\left(E_{k}+i 0\right)\right| a_{i}^{\epsilon}\right\rangle & \text { if } & i=j \\
-\left\langle a_{i}^{\epsilon}\left|R_{0}\left(E_{k}+i 0\right)\right| a_{j}^{\epsilon}\right\rangle & \text { if } & i \neq j .
\end{array}\right.
$$

The next step is to take the limit $\epsilon \longmapsto 0$, for which we need a determination of 232 the functions $\lambda_{i}(\epsilon)$ for all values of $i=1,2, \ldots, N$. This has been motivated and 233 determined in Section II in [6] and is

$$
\frac{1}{\lambda_{i}(\epsilon)}=\frac{1}{\lambda_{i}\left(M_{i}\right)}+\int_{0}^{\infty} d t K_{t+\epsilon}\left(a_{i}, a_{i}\right) e^{t M_{i}}
$$

where $K_{t}(x, y)$ is the heat kernel and $M_{i}$ is an unphysical renormalisation scale that 235 is chosen to be the energy of the bound state $E_{B}^{i}$ corresponding to the bound state 236 of the $i$-th delta [6]. This gives in the limit $\epsilon \longmapsto 0$,

$$
\psi_{k}^{+}(x)=e^{i k x}+\sum_{i, j=1}^{N}\left\langle x\left|R_{0}\left(E_{k}+i 0\right)\right| a_{i}\right\rangle\left[\Phi^{-1}\left(E_{k}+i 0\right)\right]_{i j} e^{i k a_{j}}
$$

Here,

$$
\left\langle x\left|R_{0}\left(E_{k}+i 0\right)\right| a_{i}\right\rangle=\frac{i \sqrt{k^{2}+m^{2}}}{k} e^{i k\left|x-a_{i}\right|}+\frac{1}{\pi} \int_{m}^{\infty} d \mu e^{-\mu\left|x-a_{i}\right|} \frac{\sqrt{\mu^{2}-m^{2}}}{\mu^{2}+k^{2}}
$$


and

$$
\begin{aligned}
& \Phi_{i j}\left(E_{k}+i 0\right)= \\
& =\left\{\begin{array}{cl}
-\frac{1}{\lambda\left(E_{\lambda}, E_{B}^{i}\right)}-\frac{i E_{k}}{\sqrt{E_{k}^{2}-m^{2}}} & \text { if } i=j, \\
-\frac{i E_{k}}{\sqrt{E_{k}^{2}-m^{2}}} e^{i \sqrt{E_{k}^{2}-m^{2}}\left|x-a_{j}\right|}-\frac{1}{\pi} \int_{m}^{\infty} d \mu e^{-\mu\left|x-a_{i}\right|} \frac{\sqrt{\mu^{2}-m^{2}}}{\mu^{2}+E_{k}^{2}-m^{2}} & \text { if } i \neq j,
\end{array}\right.
\end{aligned}
$$

where

$$
\begin{aligned}
& \frac{1}{\lambda\left(E_{\lambda}, E_{B}^{i}\right)} \\
& =\frac{-E_{k}}{\pi \sqrt{E_{k}^{2}-m^{2}}} \operatorname{arctanh}\left(\frac{\sqrt{E_{k}^{2}-m^{2}}}{E_{k}}\right)-\frac{E_{B}^{i}}{\pi \sqrt{m^{2}-\left(E_{B}^{i}\right)^{2}}}\left(\frac{\pi}{2}+\arcsin \frac{E_{B}^{i}}{m}\right),
\end{aligned}
$$

where $E_{B}^{i}$ has been defined before and $\mu:=\min _{i} E_{B}^{i}$. The conclusion is that the 242 Lippmann-Schwinger equation gives in a rather straightforward manner the exact 243 form of the scattering states in a rather cumbersome situation as the one discussed 244 along the present section. Explicit expressions for transmission and reflection 245 coefficients can be also derived from the above expressions.

\section{Concluding Remarks}

The Lippmann-Schwinger equation is a useful tool that permits to obtain explicit 248 forms for the scattering states produced by some potential. When this potential 249 is a finite set of Dirac delta interactions, one may find explicit expressions for 250 these scattering states. We have shown that this is the case when perturbing the 251 free Schrödinger one dimensional and the Salpeter Hamiltonians with $N$ attractive 252 deltas. In the first case, we have also shown that the Lippmann-Schwinger equation 253 gives explicit expressions for Gamow wave functions which are the wave function 254 for the purely exponential decay part of resonance states. The discussion on the 255 search for bound states for the Schrödinger case includes similar methods. 256

The one dimensional Salpeter Hamiltonian with $N$ attractive deltas is much more 257 complicated as it requires of a regularisation procedure that we implement with the 258 use of the heat kernel for the pseudo-differential operator $\sqrt{-d^{2} / d x^{2}+m^{2}}$. In this 259 case, we also obtain the exact form of the scattering states.

Acknowledgements We dedicate this paper to Professor Véronique Hussin for her contributions 261 to science and her friendship. The present work has been fully financed by TUBITAK from Turkey 262 
under the "2221 - Visiting Scientist Fellowship Programme”. We are very grateful to TUBITAK 263 for this support. We also acknowledge Osman Teoman Turgut for clarifying discussions and his 264 interest in the present research. This work was also sponsored by the Ministerio de Economía y 265 Competitividad of Spain (Project No. MTM2014-57129-C2-1-P with EU-FEDER support) and the 266 Junta de Castilla y León (Projects VA057U16, VA137G18 and BU229P18).

\section{References}

1. S. Albeverio, F. Gesztesy, R. Høeg-Krohn, H. Holden, Solvable Models in Quantum Mechanics 269 (AMS Chelsea Series, Providence RI, 2004) 270

2. Y.N. Demkov, V.N. Ostrovskii, Zero-range Potentials and Their Applications in Atomic Physics 271 (Plenum, New York, 1988) 272

3. M. Belloni, R.W. Robinett, The infinite well and Dirac delta function potentials as pedagogical, 273 mathematical and physical models in quantum mechanics. Phys. Rep. 540, 25-122 (2014) 274

4. S. Albeverio, P. Kurasov, Singular Perturbations of Differential Operators Solvable 275 Schrödinger-type Operators (Cambridge University Press, Cambridge, 2000) 276

5. M.H. Al-Hashimi, A.M. Shalaby, U.-J.Wiese, Asymptotic freedom, dimensional transmuta- 277 tion, and an infrared conformal fixed point for the $\delta$-function potential in one-dimensional 278 relativistic quantum mechanics. Phys. Rev. D 89, 125023 (2014) 279

6. F. Erman, M. Gadella, H. Uncu, One-dimensional semirelativistic Hamiltonian with multiple 280 Dirac delta potentials. Phys. Rev. D 95, 045004 (2017) 281

7. M. Calçada, J.T. Lunardi, L.A. Manzoni, W. Monteiro, Distributional approach to point 282 interactions in one-dimensional quantum mechanics. Front. Phys. 2, 23 (2014) 283

8. F. Erman, M. Gadella, S. Tunal, H. Uncu, A singular one-dimensional bound state problem 284 and its degeneracies. Eur. Phys. J. Plus 132, 352 (2017) 285

9. F. Erman, M. Gadella, H. Uncu, On scattering from the one dimensional multiple Dirac delta 286 potentials. Eur. J. Phys. 39, 035403 (2018) 287

10. R. de L. Kronig, W.G. Penney, Quantum mechanics of electrons in crystal lattices. Proc. R. 288 Soc. A 130, 499 (1931) 289

11. C. Kittel, Introduction to Solid State Physics 8th edn. (Wiley, New York, 2005) 290

12. I.R. Lapidus, Resonance scattering from a double $\delta$-function potential. Am. J. Phys. 50, 663- 291 664 (1982) 292

13. P. Senn, Threshold anomalies in one dimensional scattering. Am. J. Phys. 56, 916-921 (1988) 293

14. P.R. Berman, Transmission resonances and Bloch states for a periodic array of delta function 294 potentials. Am. J. Phys. 81, 190-201 (2013) 295

15. S.H. Patil, Quadrupolar, triple $\delta$-function potential in one dimension. Eur. J. Phys. 629-640 296 (2009)

16. V.E. Barlette, M.M. Leite, S.K. Adhikari, Integral equations of scattering in one dimension. 298 Am. J. Phys. 69, 1010-1013 (2001) 299

17. D. Lessie, J. Spadaro, One dimensional multiple scattering in quantum mechanics. Am. J. Phys. 300 54, 909-913 (1986) 301

18. J.J. Alvarez, M. Gadella, L.M. Nieto, A study of resonances in a one dimensional model with 302 singular Hamiltonian and mass jump. Int. J. Theor. Phys. 50, 2161-2169 (2011) 303

19. J.J. Alvarez, M. Gadella, L.P. Lara, F.H. Maldonado-Villamizar, Unstable quantum oscillator 304 with point interactions: Maverick resonances, antibound states and other surprises. Phys. Lett. 305 A 377, 2510-2519 (2013) 306

20. A. Bohm, in The Rigged Hilbert Space and Quantum Mechanics. Springer Lecture Notes in 307 Physics, vol. 78 (Springer, New York, 1978) 308

21. J.E. Roberts, Rigged Hilbert spaces in quantum mechanics. Commun. Math. Phys. 3, 98-119 309 (1966) 
22. J.P. Antoine, Dirac formalism and symmetry problems in quantum mechanics. I. General 311 formalism. J. Math. Phys. 10, 53-69 (1969) 312

23. O. Melsheimer, Rigged Hilbert space formalism as an extended mathematical formalism for 313 quantum systems. J. Math. Phys. 15, 902-916 (1974) 314

24. M. Gadella, F. Gómez, On the mathematical basis of the Dirac formulation of quantum 315 mechanics. Int. J. Theor. Phys. 42, 2225-2254 (2003) 316

25. A. Bohm, Quantum Mechanics. Foundations and Applications (Springer, Berlin, New York, 317 2002)

26. M.C. Fischer, B. Gutiérrez-Medina, M.G. Raizen, Observation of the quantum Zeno and anti- 319 Zeno effects in an unstable system. Phys. Rev. Lett. 87, 40402 (2001) 320

27. C. Rothe, S.L. Hintschich, A.P. Monkman, Violation of the exponential-decay law at long 321 times. Phys. Rev. Lett. 96, 163601 (2006) 322

28. A. Bohm, Resonance poles and Gamow vectors in the rigged Hilbert space formulation of 323 quantum mechanics. J. Math. Phys. 22 (12), 2813-2823 (1981) 324

29. A. Bohm, M. Gadella, in Dirac Kets, Gamow Vectors and Gelfand Triplets. Springer Lecture 325 Notes in Physics, vol. 348 (Springer, Berlin, 1989) 326

30. O. Civitarese, M. Gadella, Physical and mathematical aspects of Gamow states. Phys. Rep. 327 396, 41-113 (2004) 328

31. O. Civitarese, M. Gadella, Gamow states as solutions of a modified Lippmann-Schwinger 329 equation. Int. J. Mod. Phys. E 25, 1650075 (2016)

32. M. Reed, B. Simon, Analysis of Operators (Academic, New York, 1978), p. 55

33. M. Gadella, F. Gómez, The Lippmann-Schwinger equations in the rigged Hilbert space. J. 332 Phys. A: Math. Gen. 35, 8505-8511 (2002) 333

34. T. Berggren, Expectation value of an operator in a resonant state. Phys. Lett. B 373, 1-4 (1996) 334

35. O. Civitarese, M. Gadella, R. Id Betan, On the mean value of the energy for resonance states. 335 Nucl. Phys. A 660, 255-266 (1999) 